\title{
Segmentation of Liver from Abdomen CT images and 3D Visualization
}

\author{
${ }^{1}$ Ritambhara and ${ }^{2}$ Deepti Mittal \\ Department of Electrical and instrumentation Engineering \\ Thapar University, Patiala, Punjab, India \\ ${ }^{1}$ ritambhara3939@gmail.com; ${ }^{2}$ deepti.mittal@thapar.edu
}

\begin{abstract}
Segmentation of liver is the initial and fundamental step for the diagnosis of liver disease, 3-D volume construction and volume measurement. However, segmentation of liver is a challenging task due to it's inter and intra intensity and texture similarities among other organs present in CT abdominal images. A semiautomatic method has been proposed to segment the liver portion from CT abdominal images and their three dimensional volume construction by (i)Noise removal using median filter,(ii)Segmentation of liver portion based on active contour method using sparse field method and(iii)liver volume construction using marching cube method. Evaluation of proposed method is carried out on clinically acquired CT images and effectiveness of algorithm is evaluated by comparing manually segmented liver portion marked by radiologist with proposed method.
\end{abstract}

Keywords-Active contour, marching cube.

\section{Introduction}

Computed Tomography (CT) is one of the imaging technique that is used to obtain cross-sectional image of soft tissues inside the body. Each cross-sectional image is termed as a "slice" of the person being imaged. These cross-sectional images are used to diagnose liver diseases and for construction of three dimensional volume of liver. There are number of imaging techniques to diagnose liver diseases like ultrasound, computed tomography and magnetic resonance imaging (MRI). Ultrasound is least expensive and most available imaging technique but it is operator dependent and does not give good quality and contrast of image based on echo genecity. Although MRI gives better contrast and is highly specific, it is very expensive and only available at specialized units. Therefore it is not as popular as CT. Computed tomography is comparatively less costly than MRI and gives better contrast than ultrasound. Therefore it is frequently applied imaging technique.

Liver is the largest organ in the body and performs many vital functions. According to American Cancer Society, liver cancer is the sixth most common cancer in the world [1]. Major cause of primary cancer is alcohol-related cirrhosis and fatty liver diseases. Fatty liver diseases are those liver diseases in which fat accumulates on the liver usually in connection with heavy use of alcohol and weight gain which increase volume of liver. Cirrhosis is end stage of liver cancer with reduced liver volume. Therefore volume measurement may be required by the radiologist to diagnose the conditions of such type of liver diseases. 
Ritambhara and Deepti Mittal; Segmentation of Liver from Abdomen CT images and 3D Visualization. Journal of Biomedical Engineering and Medical Imaging, Volume 2, No 5, October (2015) , pp 46-55

Segmentation of liver is a fundamental preprocessing step for many applications such as liver tumor segmentation, 3-D (three dimensional) volume construction and volume measurement. The segmentation of liver portion is primarily done to diagnose liver tumor which is main cause of liver cancer. Another major advantage of segmentation of liver is three dimensional volume construction of liver. In three dimensional volume constructions, segmented liver slices are stacked to form a volume. 3-D volume is vitally used in tumor resection, transplantation of liver and in invasive surgery. 3-D volume construction will assist radiologist for the confirmation of liver diseases like cirrhosis and fatty liver diseases based on variation of liver volume as described above. Major challenge in 3-D modeling is determination of correspondence between segmented instances. The quality of model, completeness and accuracy are main aspects which are to be considering in modeling of 3-D volume. In this preliminary work, a method has been proposed which is designed by considering effective segmentation of liver portion from CT images. In order to develop an efficient 3-D method, an effective segmentation method is needed to be applied on the clinically acquired medical images. That's why a huge literature review has been carried out.

Ananth et al. developed a method to segment the liver in which they compare two methods Graph cut and geodesic graph cut method [2]. They achieved Dice similarity coefficient (DSC) $=87.1 \%$, processing time $=1.005 \mathrm{~s}$ in case of graph cut method and DSC $=94.5 \%$, processing time $=1.009 \mathrm{~s}$ in case of geodesic graph cut method. Main advantage of this method is to precisely localize object boundaries. Kumar et al. developed an automatic method in which they used morphological functions (erosion) in pre-processing to reduce computation time and efforts by removing extra regions and segmentation of liver is done using region growing method [3]. The main drawback of region growing method is manual seed point identification. If this seed point is not located properly, it can lead to false segmentation. Jayanthi and Kanmani developed segmentation method of region growing with morphological functions in post processing step [4]. They analyze that for entire region segmentation, region growing method has to be applied in iterative manner. Mittal and Kumari used gradient based segmentation method to find true edges in their work [5]. Lim et al. developed automatic liver segmentation method using adaptive thresholding and iterative morphological filtering [6]. They obtained an accuracy of $96 \%$. Applied method took 2-3 minutes for total processing. A survey of different segmentation techniques are given by Rajagopal and Subbaiah [7]. They gave comparative study of region growing and thresholding based segmentation methods. They analyze that region growing methods are best employed on contrast enhanced CT images and threshold based approaches best work on the images in which intensity histogram gives neat intensity peak for input image. Massieh et al. developed a method in which they used isodata thresholding technique and Mean shift filter in preprocessing and force-driven optimized active contour (snake) to segment liver portion. It gives accuracy across $95 \%$. This method can be further used to segment tumor portion in liver. Main limitation of the method is that snake does not stop at the boundary of liver [8]. Other limitation is that in some cases the boundary between liver and neighboring organs disappears and it becomes hard to determine the difference among different organs and this leads to erroneous segmentation. Casciaro et al. gives an adaptive initialization method to produce fully automatic processing frameworks based on graph-cut and gradient flow active contour algorithms [9]. They used mean shift filter for pre-processing because mean shift filter preserve edges and does not blur edges. In their study graph-cut algorithm and active contour gradient vector flow gives DSC $=95.49 \%$ and $96.17 \%$ respectively. In above defined method preprocessing step consumes $70 \%$ of total processing time. 
Massoptier and Casciaro developed a method in which they used mean shift filter in preprocessing step in which designed filter reduce pre-processing time by $90 \%$ and were combined to an active contour technique using gradient vector flow in order to obtain smoother segmentation [10]. A 3-D stastical model was constructed to best define the shape of liver. Elaziz et al. developed a method based on regiongrowing [11]. They claim that their algorithm decreases the computation time by removing the regions of other structures as most techniques in use today are very time consuming. A lot of work has been done on CT images [12][13].

Brief literature review of recent development is shown in Table1. In literature review, authors states that mean shift filter consume most of the processing time therefore a method is designed in which median filter is used. It leads comparatively less time with not much deterioration in segmented result. It will be further helpful in real time implementation of the proposed method.

Table1.Summary of previous research work related to segmentation of liver and construction of 3D volume

\begin{tabular}{|c|c|c|c|c|c|c|c|}
\hline Method & Year & $\begin{array}{c}\text { Dataset } \\
\text { (CTimage) }\end{array}$ & $\begin{array}{l}\text { Image processing } \\
\text { technique } \\
\text { (preprocessing) }\end{array}$ & $\begin{array}{l}\text { Image processing } \\
\text { technique } \\
\text { (segmentation) }\end{array}$ & Parameter & Pros & Cons \\
\hline $\begin{array}{l}\text { Lim et } a l . \\
{[6]}\end{array}$ & 2005 & - & Thresholding based & $\begin{array}{l}\text { Contour method } \\
\text { based on } \\
\text { morphological } \\
\text { filtering }\end{array}$ & $A C=96 \%$ & $\begin{array}{l}\text { Preprocessing steps } \\
\text { are more. } \\
\text { Processing time 20- } \\
\text { 60s/slice. }\end{array}$ & $\begin{array}{l}\text { Total processing time } \\
\text { is } 2-3 \text { minutes. }\end{array}$ \\
\hline $\begin{array}{c}\text { Massoptier } \\
\text { and Casciaro } \\
{[10]}\end{array}$ & 2008 & 21 & $\begin{array}{c}\text { Mean shift filter } \\
\text { based }\end{array}$ & $\begin{array}{l}\text { Active contour } \\
\text { using gradient } \\
\text { vector flow }\end{array}$ & $\begin{array}{l}\mathrm{VO}=94.2 \% \\
\mathrm{Ac}=3.7 \mathrm{~mm}\end{array}$ & $\begin{array}{c}\text { GVF active contour } \\
\text { converge faster than } \\
\text { active contour. }\end{array}$ & $\begin{array}{l}\text { Designed filter save } \\
90 \% \text { of processing } \\
\text { time. }\end{array}$ \\
\hline $\begin{array}{c}\text { Campadelli et } \\
\text { al.[14] }\end{array}$ & 2009 & 100 & $\begin{array}{l}\text { Images resized to } \\
256 \times 256 \text { pixels, } 3 \times 3 \\
\text { median filter based. }\end{array}$ & $\begin{array}{l}\text { Gray level based } \\
\text { framework }\end{array}$ & SVO $=94 \%$ & $\begin{array}{l}\text { Down sampling } \\
\text { factor reduce } \\
\text { computation cost. }\end{array}$ & - \\
\hline $\begin{array}{c}\text { Moghe et al. } \\
\text { [15] }\end{array}$ & 2011 & 58 & No preprocessing & $\begin{array}{c}\text { Automatic } \\
\text { thresholding }\end{array}$ & - & $\begin{array}{c}\text { If Intensity variation } \\
\text { is sharp. }\end{array}$ & - \\
\hline $\begin{array}{c}\text { Massieh et al. } \\
{[8]}\end{array}$ & 2011 & 15 & $\begin{array}{c}\text { Isodata thresholding } \\
\text { and Mean shift filter } \\
\text { based }\end{array}$ & $\begin{array}{c}\text { Force-driven } \\
\text { optimized active } \\
\text { contour (snake) }\end{array}$ & $\begin{array}{l}\mathrm{Se}=95 \% \\
\mathrm{Sp}=99 \%\end{array}$ & $\begin{array}{l}\text { Method can be used } \\
\text { further for } \\
\text { diagnosing the } \\
\text { lesions. }\end{array}$ & $\begin{array}{c}\text { Snake does not stop } \\
\text { at the Boundary of } \\
\text { liver. }\end{array}$ \\
\hline $\begin{array}{c}\text { Casciaro et al. } \\
\text { [9] }\end{array}$ & 2012 & 10 & Mean shift filter & $\begin{array}{l}\text { Graph-cut } \\
\text { algorithms }\end{array}$ & $\mathrm{DSC}=96.2 \%$ & High accuracy & $\begin{array}{c}\text { Filtering consume } \\
70 \% \text { of process time. }\end{array}$ \\
\hline $\begin{array}{c}\text { Ananth et al. } \\
\text { [2] }\end{array}$ & 2014 & - & Mean shift filter & $\begin{array}{c}\text { Geodesic Graph cut } \\
\text { method. }\end{array}$ & $\begin{array}{l}\mathrm{DSC}=94.5 \% \\
\mathrm{PT}=1.009 \mathrm{~s}\end{array}$ & $\begin{array}{c}\text { Geodesic graph cut } \\
\text { is better }\end{array}$ & - \\
\hline
\end{tabular}

${ }^{*} A C=$ average correctness, $V O=$ volume overlap, $D S C=$ Dice similarity coefficient, $P T=$ processing time, Se=sensitivity, $S p=s p e c i f i c i t y$, $S V O=$ Symmetric volume overlap, $A c=$ Accuracy

\section{Materials and methods}

The proposed method is applied on a data set containing 75 CT images. CT images are of $514 \times 514$ dimensions. The data set of CT images is collected from the MAX hospital, Delhi, India. The method is implemented in MATLAB version 8.The block diagram representation of the proposed is shown in Fig.1, 
Ritambhara and Deepti Mittal; Segmentation of Liver from Abdomen CT images and 3D Visualization. Journal of Biomedical Engineering and Medical Imaging, Volume 2, No 5, October (2015) , pp 46-55

where it is expressed in terms of three major steps such as: preprocessing, segmentation of liver portion using active contour method and 3D volume construction.

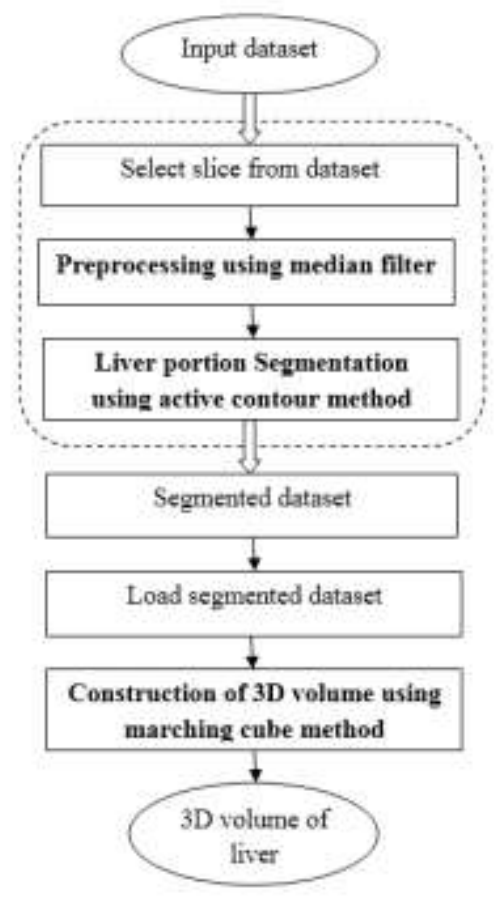

Figure 1 Block diagram representation of proposed methodology for 3D volume construction

\subsection{Preprocessing}

CT images may be noisy because of its making procedure, data acquisition and construction of image from the acquired data. Also, near boundary portion of the CT images contain patient related information along with other information. Therefore it is ethically necessary to hide that information in each CT image. Therefore cropping is applied as initial step of preprocessing and after that median filter is applied for the removal of noise dataset. Applied method uses resized CT image slices in which slices of $514 \times 514$ dimensions are reduced to $307 \times 457$ dimensions. This step removes all text information which is not necessary for processing and reduces processing time.

Median filter of size $3 \times 3$ is convolved with CT image. The noise removal in an image is necessary for effective segmentation; at the same time it is important to preserve the edges. Edges in CT image are of critical importance for the visual appearance of images. The median filter is demonstrably better than Gaussian blur for removing noise whilst preserving edges for a given, fixed window size for moderate level noise (Gaussian).

\subsection{Liver segmentation using Active contour method}

In this work, active contour method is used to segment liver portion from the clinically acquired abdominal CT images. Active contour method finds the curve that best separates objects in an image [16]. Active contour or snakes is an energy minimizing spline guided by external constraint forces and pulled by image forces toward features like edge detection, subjective contours, motion tracking etc. Snakes falls into local energy minima for which they rely on other mechanisms to place them near the desired contour. Snakes 
will try to match a deformable model to an image by means of energy minimization. Total energy of snakes is given by

$$
E_{\text {snakes }}=\int_{0}^{1}\left(E_{\text {int }}(b(x))+E_{\text {im }}(b(x))+E_{\text {con }}(b(x))\right) d x
$$

$\mathrm{E}_{\mathrm{int}}=$ Internal energy due to bending(elasticity)

$\mathrm{E}_{\mathrm{im}}=$ Image forces pushing the snake toward image features

$\mathrm{E}_{\mathrm{con}}=$ External constraints put the snakes near the desired local minima

Internal energy of snake is composed of two parameters elasticity and rigidity which can be expressed by

$$
\left.\mathrm{E}_{\mathrm{int}}=\int_{l}\left(\frac{1}{2} \alpha(\mathrm{x})|\mathrm{b}(\mathrm{x})|^{2}+\frac{1}{2} \beta(\mathrm{x})|\mathrm{b}(\mathrm{x})|^{2}\right)\right) \mathrm{dx}
$$

$\alpha(\mathrm{x}), \beta(\mathrm{x})$ control the amount of stretch in the snake and the amount of curvature in the snake respectively. Image energy is a function of features of the image. I(x,y) image is a combination of lines, edges and termination. These can be expressed as

$$
\mathrm{E}_{\text {image }}=\mathrm{w}_{\text {line }} \mathrm{E}_{\text {line }}+\mathrm{w}_{\text {edge }} \mathrm{E}_{\text {edge }}+\mathrm{w}_{\text {term }} \mathrm{E}_{\text {term }}
$$

Here $\mathrm{w}_{\text {line }}, \mathrm{w}_{\text {edge }}, \mathrm{w}_{\text {term }}$ are weights of features, higher values of feature will indicate higher contribution of that salient feature.

Line functional is the intensity of image and is given by

$$
\mathrm{E}_{\text {line }}=\mathrm{I}(\mathrm{x}, \mathrm{y})
$$

Edge functional will attract the snake to large intensity gradient

$$
\mathrm{E}_{\text {edge }}=-|\nabla \mathrm{I}(\mathrm{x}, \mathrm{y})|^{2}
$$

Termination functional will attract the snake towards termination of line segment or corner. Constraint energy will guide the snake to go away or towards a particular feature. Proposed method given by Kass et al.[17] has various limitations therefore many researchers had improved the active contour method by introducing various models based on edge and region functional. One such model is geometric or geodesic snake model based on Euclidian curve shortening evolution. These models are implemented using level sets. Level sets have a limitation of slow computation. This limitation is overcome by sparse field method (SFM) introduced by R.Whitaker [18]. Sparse field method drastically reduces computation times for level set methods. The initial contour is chosen to include the object. The sparse field method uses lists of points that are composed of zero level set as well as neighboring points to the zero level set. A very efficient representation of contour is done by using these lists and carefully moving points to and from the appropriate list. To minimize some segmentation energy level sets are deformed. There are many such active contour energies. Most popular is Chan-Vese energy [19]

$$
E=\int_{\text {interior }}(I-\mu)^{2}+\int_{\text {exterior }}(I-\mu)^{2}
$$

In SFM the speed of curve updates is dependent only on the length of the curve, and not on the size of the image. The active contour will stop its iterations when recent five iterations give the same result. 
Ritambhara and Deepti Mittal; Segmentation of Liver from Abdomen CT images and 3D Visualization. Journal of Biomedical Engineering and Medical Imaging, Volume 2, No 5, October (2015) , pp 46-55

\subsection{Three Dimensional Volume Construction}

The final step in proposed methodology is 3-D volume construction from 2-D images. That will be primarily used for visualization and quantization of liver. Volume construction of CT images will reduce inter observer variability by correctly evaluating the size of liver which is irregular in shape and size. Various methods have been introduced to construct stastical shape models or 3-D volume [20]. Heimann et al. had given a detailed review of all the stastical shape models based upon alignment, correspondence, registration etc. In this paper marching cube method is used to display overall volume of CT 2-D images. One such efficient method for volume construction is Marching cube method. It was introduced by Lorensen and Cline in 1987 [21].

Marching cubes algorithm proceeds through the voxles or scalar field. It takes eight neighbor locations at a time. Therefore these points form an imaginary cube. Then polygon(s) is determined which will be used to represent the part of the isosurface that will pass through this cube. The individual polygons are then combined to form a desired three dimensional surface. This whole process is done by creating an index, since there are eight vertices of cube and two neighbor slices; therefore there are total 256 configurations within the cube. If value of one of the vertices in the cube is higher than the iso-value that means that point is inside the surface therefore that particular bit is set to one. Otherwise if value of vertices is lower than iso-value, it is set to zero. Finally generated polygons are placed at appropriate location by linear interpolation. In this way 3-D volume surface is acquired using Marching cube method.

\section{Experimental results and discussion}

The proposed methodology is evaluated visually and quantitatively, analysis is done on CT images. In the first step of the method segmentation of liver is carried out on the acquired CT images using active contour method. These results of segmentation are demonstrated in fig. 2 on an example image.

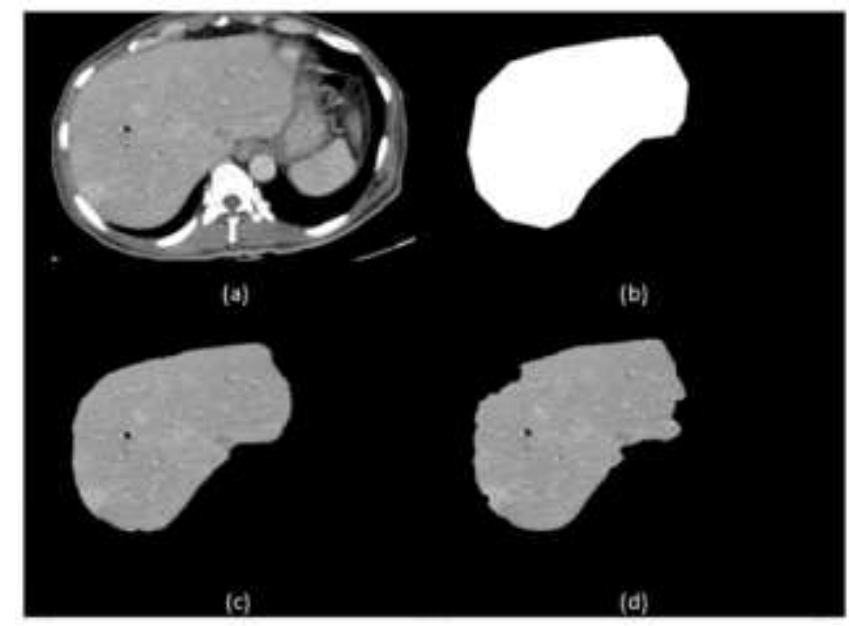

Figure 2 Liver portion segmentation result (a) 307x457 resized Original image (b) Initial mask (c) Segmented image with 50 iterations (d) Segmented image with 200 iterations

Figure 2 (a) is a resized image of dimension $307 \times 457$. Noise in clinically acquired CT image is removed using median filter and it will take less time for preprocessing. Active contour method was applied to segment liver region. Fig.2 (b) is a mask obtained by initializing points on CT image by prior knowledge of position and shape of liver. The points should be marked at the boundary of liver region for accurate segmentation. Active contour method uses 'Chan-vese'or 'edge' method to minimize its segmentation energy and to 
extract edge features from the image. 'Chan-vese' cannot correctly distinguish the edges when the intensity variation is not so sharp. Therefore 'edge' functional is used to detect the edges in CT images. Segmentation method will stop iterate when previous five iterations give same result. Fig.2(c) shows segmentation result with maximum 50 iterations. 'Edge' method has a natural tendency to shrink at the boundary of the region with increase in number of iterations. During segmentation it is essential to initialize the points outside the boundary of region of interest. Therefore a threshold is essential to set, otherwise image will get deteriorate. Fig 2(d) shows segmented CT image after 200 iterations. From fig. 2 (d) the need of thresholding can be easily visualized where image got deteriorate at the boundary of liver portion due to increase in number of iterations.

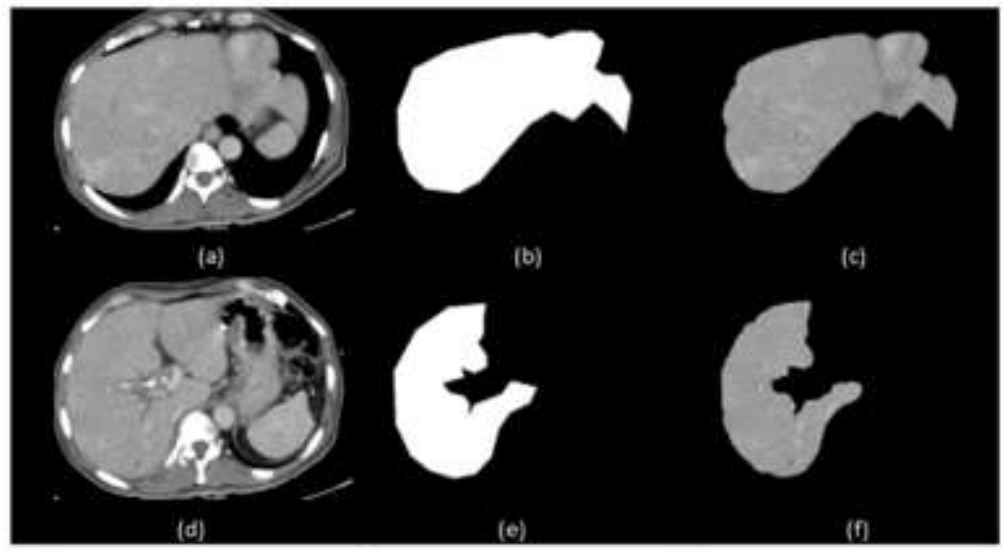

Figure 3 from top to bottom: $\mathrm{CT}$ images with different difficulty cases in CT dataset.

Figure 3 shows the segmentation results on different samples of CT images where difficulty level is comparatively high. From top to bottom in Fig. 3 it is clearly visualized that active contour method can efficiently segment liver portion from CT images. Quantative analysis of active contour method is carried out by shape feature extraction. Table 2 shows the result of shape feature extraction. Area tells us about the actual number of pixel in segmented region. Perimeter is the total distance around the boundary of 2D slice. Equiv-diameter gives diameter of region of interest. Table 2 shows the result of clinically acquired manually segmented liver CT images by radiologist. Table 2 shows the result of active contour method applied on CT images. Relative accuracy is calculated by subtraction of manually segmented result by radiologist and active contour method divided by manually segmented result.

$$
\text { Relative accuracy }=\frac{\text { manually segmented result by radiologist-active contour method result }}{\text { manually segmented result by radiologist }}
$$

Comparative performance analysis shows the comparison of results of manually segmented CT images by radiologist and segmented liver portion results using active contour method in Table2. In this preliminary work on 2-D images, 3-D volume is constructed using marching cube method. In first step, segmented liver dataset is loaded. Fig. 4 is showing whole process of construction of 3-D volume. 3-D volume will give a better visualization of all CT image slices 
Ritambhara and Deepti Mittal; Segmentation of Liver from Abdomen CT images and 3D Visualization. Journal of Biomedical Engineering and Medical Imaging, Volume 2, No 5, October (2015) , pp 46-55

Table2: Comparison between manually segmented image result and active contour method result

\begin{tabular}{|c|c|c|c|c|c|c|c|c|c|}
\hline \multirow[t]{2}{*}{$\begin{array}{c}\mathrm{CT} \\
\text { images }\end{array}$} & \multicolumn{3}{|c|}{$\begin{array}{c}\text { Area } \\
\text { (pixels) }\end{array}$} & \multicolumn{3}{|c|}{ Perimeter } & \multicolumn{3}{|c|}{ Equiv-diameter } \\
\hline & $\begin{array}{l}\text { Manually } \\
\text { segmented } \\
\text { liver } \\
\text { portion }\end{array}$ & $\begin{array}{c}\text { Segmented } \\
\text { liver portion } \\
\text { by proposed } \\
\text { method }\end{array}$ & $\begin{array}{l}\text { Relative } \\
\text { accuracy }\end{array}$ & $\begin{array}{l}\text { Manually } \\
\text { segmented } \\
\text { liver } \\
\text { portion }\end{array}$ & $\begin{array}{c}\text { Segmented } \\
\text { liver portion } \\
\text { by proposed } \\
\text { method }\end{array}$ & $\begin{array}{l}\text { Relative } \\
\text { accuracy }\end{array}$ & $\begin{array}{c}\text { Manually } \\
\text { segmented } \\
\text { liver } \\
\text { portion }\end{array}$ & $\begin{array}{c}\text { Segmented } \\
\text { liver portion } \\
\text { by proposed } \\
\text { method }\end{array}$ & $\begin{array}{l}\text { Relative } \\
\text { accuracy }\end{array}$ \\
\hline Slice 1 & 45982 & 43724 & 0.0491 & 875.09 & 823.08 & 0.0591 & 241.88 & 235.847 & 0.0249 \\
\hline Slice 2 & 45926 & 43155 & 0.0603 & 901.62 & 867.56 & 0.0377 & 241.73 & 234.306 & 0.0177 \\
\hline Slice 3 & 46777 & 44320 & 0.0525 & 874.59 & 838.16 & 0.0416 & 243.96 & 237.450 & 0.0266 \\
\hline Slice 4 & 46274 & 45120 & 0.0249 & 882.60 & 850.53 & 0.0363 & 242.64 & 239.580 & 0.0129 \\
\hline Slice 5 & 42608 & 43981 & 0.0322 & 1248.60 & 860.90 & 0.3105 & 232.79 & 236.540 & 0.1608 \\
\hline Slice 6 & 40217 & 37583 & 0.0654 & 884.11 & 1223.70 & 0.3830 & 226.15 & 218.590 & 0.0334 \\
\hline Slice 7 & 29019 & 27385 & 0.0560 & 960.98 & 929.81 & 0.0324 & 192.09 & 186.570 & 0.0287 \\
\hline Slice 8 & 28716 & 26492 & 0.0774 & 1026.00 & 954.94 & 0.0694 & 191.09 & 183.500 & 0.0397 \\
\hline Slice 9 & 28523 & 26095 & 0.0851 & 981.07 & 956.49 & 0.0250 & 190.44 & 182.124 & 0.0461 \\
\hline Slice 10 & 26712 & 25840 & 0.0321 & 1007.60 & 943.16 & 0.0639 & 184.27 & 181.244 & 0.0164 \\
\hline
\end{tabular}

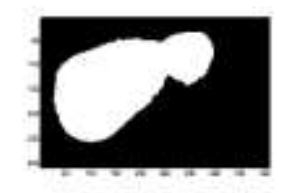

(a)

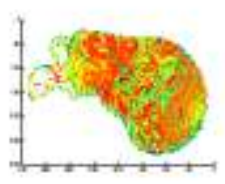

(c)

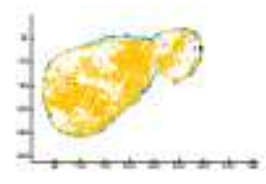

(b)

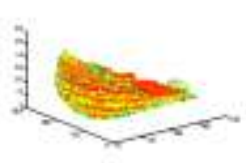

(d)

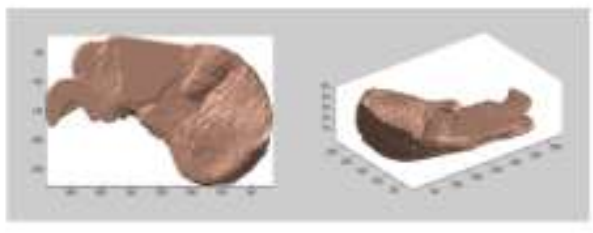

(e)

(f)

Figure 4. 3D volume construction steps: (a) 2D slice from CT dataset (b) contour slice (c) 3D contour slices stacked from 1 to 51 and its volume at 45 degree rotation (d) 3D volume of 51 slices at 90 degree rotation.

From fig. 4 it can be clearly visualized that using marching cube method 3-D volume can be constructed. Fig.4 (a) shows one of 2-D slice from dataset. Fig. 4(b) shows 2-D contour of slice.fig 4(c) is the 3-D visualization of various slices stacked together .fig $4(\mathrm{~d})$ is the smoothed 3-D model of liver.

\section{Conclusion and future scope}

A methodology is designed in the proposed work to construct 3-D volume of the liver using abdominal CT images. The methodology can be described in three main steps: (i) Noise removal (ii) segmentation of liver 
potion from abdominal CT images (iii) 3-D volume construction. The removal of noise from CT images is carried out by median filtering because of its simplicity and computational time than other filtering techniques. Segmentation of liver portion from CT images is carried out using active contour method and 3-D volume construction is done using marching cube method.

In future work this semiautomatic method can be improved by making it fully automatic to avoid user interaction. Furthermore, 3-D model that is constructed by using segmented 2-D images has certain irregularities and is not smooth. Therefore the future research work can be focused on the improvement of smoothness of model and its parameterization.

\section{REFERENCES}

[1] Corporate Center: American Cancer Society Inc. 250 Williams Street, NW, Atlanta, GA 303031002 (404) 320-3333.

[2] C. Ananth, D.L.R. Bai , K. Renuka, C. Savithra, A. Vidhya, Interactive Automatic Hepatic Tumor CT Image Segmentation, International Journal of Emerging Research in Management \&Technology ISSN: 2278-9359 (Volume-3, Issue-1),2014.

[3] S.S. Kumar, R.S. Moni, J. Rajeesh, Automatic Segmentation of Liver and Tumor for CAD of Liver, Journal of advances in information technology, vol. 2, no. 1, February 2011.

[4] M. Jayanthi and B. Kanmani, Extracting the Liver and Tumor from Abdominal CT Images, 2014 Fifth International Conference on Signals and Image Processing 978-0-7695-5100-5/13\$31.00 (C) 2013 IEEE.

[5] D. Mittal and K. Kumari, Automated detection and segmentation of drusen in retina fundus images, Computers and Electrical Engineering 47 (2015) 82-95.

[6] S.-J. Lim, Y.-Y. Jeong, Y.-S. Ho, Automatic liver segmentation for volume measurement in CT images, 1047-3203/\$-see front matter 2005 Elsevier Inc.

[7] R. Rajagopal and P. Subbaiah, A survey on liver tumor detection and segmentation methods, ARPN Journal of Engineering and Applied Sciences Vol. 10, NO. 6, April 2015.

[8] N.H. Abdel-massieh, M.M. Hadhoud, and K.A. Moustafa, A fully automatic and efficient technique for liver segmentation from abdominal CT images, presented at Informatics and Systems (INFOS), 2010 The 7th International Conference on, 2010.

[9] S. Casciaro, R. Franchini, L. Massoptier, E. Casciaro, F. Conversano,A. Malvasi and A. LayEkuakille, Fully Automatic Segmentations of Liver and Hepatic Tumors From 3-D Computed Tomography Abdominal Images: Comparative Evaluation of Two Automatic Methods, IEEE Sensors journal, vol. 12, no. 3, March 2012. 
Ritambhara and Deepti Mittal; Segmentation of Liver from Abdomen CT images and 3D Visualization. Journal of Biomedical Engineering and Medical Imaging, Volume 2, No 5, October (2015) , pp 46-55

[10] L. Massoptier, S. Casciaro, A new fully automatic and robust algorithm for fast segmentation of liver tissue and tumors from CT scans, Euro Radial (2008).

[11] O.F. Abd-Elaziz, M.S. Sayed and M.I. Abdullah, Liver Tumors Segmentation from Abdominal CT Images using Region Growing and Morphological Processing, 978-1-4799-5807-8/14/\$31.00 @2014 IEEE.

[12] K. Sharma and D. Mittal, Contrast Enhancement Technique for CT Images, Journal of Biomedical Engineering and Medical Imaging, Volume 2, No 1, Feb (2015) , pp 44-50.

[13] A. Krishan, D. Mittal, International Journal on Recent Technologies in Mechanical and Electrical Engineering (IJRMEE), Volume: 2 Issue: 5, May 2015.

[14] P. Campadelli, E. Casiraghi, S. Pratissoli and G. Lombardi, Automatic Abdominal Organ Segmentation from CT images, Electronic Letters on Computer Vision and Image Analysis 8(1):114, 2009.

[15] A.A. Moghe, J. Singhai, S.C Shrivastava, Automatic Threshold based Liver Lesion Segmentation in Abdominal 2D-CT Images, International Journal of Image Processing (IJIP), Volume (5): Issue (2): 2011.

[16] V. Caselles, R. Kimmel, G. Sapiro, Geodesic active contours, International Journal of Computer Vision, Volume 22, Issue 1, pp. 61-79, 1997.

[17] M. Kass, Witkin and Terzopolous, Snakes: Active contour models, International Journal of Computer Vision, pages 321-331, 1987.

[18] R. T. Whitaker, A level-set approach to 3D reconstruction from range data, International Journal of Computer Vision, Volume 29, Issue 3, pp.203-231, 1998.

[19] T.F. Chan and L.A. Vese, Active contours without edge, IEEE Transactions on Image Processing, Volume 10, Issue 2, pp. 266-277, 2001.

[20] T. Heimann and H.P. Meinzer, Statistical shape models for 3D medical image segmentation: A review, Medical Image Analysis 13 (2009) 543-563, 1361-8415/\$.

[21] W. E. Lorensen and H.E. Cline, Marching cubes: A high resolution 3D surface construction algorithm, Computer Graphics, Volume 21, Number 4, July 1987. 